\title{
The generalized Drazin inverse of operator matrices
}

\author{
Li Guo*1,2 (D), Honglin Zou ${ }^{3}$ (D), Jianlong Chen $^{1}$ \\ ${ }^{1}$ Southeast University, School of Mathematics, Nanjing, Jangsu 210096, P.R. China \\ ${ }^{2}$ Beihua University, School of Mathematics and Statistics, Jilin, Jilin 132013, P.R. China \\ ${ }^{3}$ Hubei Normal University, School of Mathematics and Statistics, Huangshi, Hubei 435002, P.R. China
}

\begin{abstract}
Representations for the generalized Drazin inverse of an operator matrix $\left(\begin{array}{cc}A & B \\ C & D\end{array}\right)$ are presented in terms of $A, B, C, D$ and the generalized Drazin inverses of $A, D$, under the condition that $B D^{d}=0$, and $B D^{i} C=0$, for any nonnegative integer $i$. Using the representation, we give a new additive result of the generalized Drazin inverse for two bounded linear operators $P, Q \in \mathbf{B}(X)$ with $P Q^{d}=0$ and $P Q^{i} P=0$, for any integer $i \geq 1$. As corollaries, several well-known results are generalized.
\end{abstract}

Mathematics Subject Classification (2010). 15A09, 47A05

Keywords. Banach space, generalized Drazin inverse, operator matrix

\section{Introduction}

Let $X$ and $Y$ be complex Banach spaces. Denote by $\mathbf{B}(X, Y)$ the set of all bounded linear operators from $X$ into $Y$ and abbreviate $\mathbf{B}(X, X)$ to $\mathbf{B}(X)$. An operator $A \in \mathbf{B}(X)$ is said to be generalized Drazin invertible if there exists an operator $A^{d} \in \mathbf{B}(X)$ such that

$$
A A^{d}=A^{d} A, \quad A^{d} A A^{d}=A^{d}, \quad A-A^{2} A^{d} \text { is quasi-nilpotent. }
$$

An operator $A \in \mathbf{B}(X)$ is called quasi-nilpotent if the spectrum $\sigma(A)=\{0\}$.

The Drazin inverse is first studied by Drazin [19] in associative rings and semigroups. The generalized Drazin inverse is investigated for rings by Harte [21-23] and for Banach algebras by Koliha [27]. The Drazin inverses and the generalized Drazin inverses for bounded linear operators on Banach spaces, especially for block matrices, have drawn a lot of discussion due to their interesting properties and wide applications [1-3,10].

Finding an explicit representation for the generalized Drazin inverse of an operator matrix $M=\left(\begin{array}{cc}A & B \\ C & D\end{array}\right)$ in terms of $A, B, C, D$ and related generalized Drazin inverses has been studied by several authors $[4,5,9,11-16,26,32,33,36,37]$. Djordjević and Stanimirović [16] generalize the well-known result in [24,31] concerning the Drazin inverse of block $2 \times 2$ upper triangular matrices to the generalized Drazin inverse for block triangular operator matrices, and further consider the case that $B C=0, B D=0$ and $D C=0$. These

\footnotetext{
*Corresponding Author.

Email addresses: guomingli95@163.com (L. Guo), honglinzou@163.com (H. Zou), jlchen@seu.edu.cn (J. Chen)

Received: 12.03.2018; Accepted: 26.08.2019
} 
requirements are relaxed and new conditions are presented in $[4,5,9,12-14]$, for example, the condition $A B C=0$ is dealt with in $[4,5,14]$ under some extra assumptions.

This paper is inspired by $[4,14,18]$. Dopazo and Matinez-Serrano [18] gave an explicit expression for the Drazin inverse of $2 \times 2$ complex block matrix $M$ under the condition that $B D^{2}=0$ and $B D^{i} C=0, i=0,1$. The results in [18] is generalized in [20] by considering more general condition that $B D^{i} C=0$, for any nonnegative integer $i$.

In this paper, we give the explicit representation for the generalized Drazin inverse of a $2 \times 2$ operator matrix $M$ under the condition that $B D^{d}=0, B D^{i} C=0$, for any nonnegative integer $i$.

Formulas for the generalized Drazin inverse of a $2 \times 2$ operator matrix can be very useful for deriving formulas for the generalized Drazin inverse of the sum of two generalized Drazin invertible elements.

Actually, In 1958, Drazin [19] first studied the representation for the Drazin inverse of the sum of two Drazin invertible elements in a ring and proved that $(a+b)^{d}=a^{d}+b^{d}$ under the condition $a b=b a=0$. Later, Koliha [27] gave the representations of $(a+b)^{d}$ under the same condition in a Banach algebra. In 2001, Hartwig, Wang and Wei [25] gave the formula $(P+Q)^{d}$ under the condition $P Q=0$. Djordjevic and Wei [7] generalized the result of [25] to bounded linear operators on an arbitrary complex Banach space. More results on generalized Drazin inverse can be found in $[6,8,29,30,35]$. In Section 4 , we give a new additive result of the generalized Drazin inverse for two bounded linear operators $P, Q \in \mathbf{B}(X)$ with $P Q^{d}=0$ and $P Q^{i} P=0$, for any integer $i \geq 1$. As corollaries, many results in $[4,5,9,13,14,16,18]$ are generalized.

\section{Preliminary}

Throughout this paper, unless otherwise stated we will make the following assumption:

$$
M=\left(\begin{array}{cc}
A & B \\
C & D
\end{array}\right)
$$

where $A \in \mathbf{B}(X), D \in \mathbf{B}(Y), B \in \mathbf{B}(Y, X)$ and $C \in \mathbf{B}(X, Y)$.

We write $\sigma(A)$ and $\rho(A)$ for the spectrum and the resolvent set of $A$, respectively. For $\lambda \in \rho(A)$, we denote the resolvent $(\lambda I-A)^{-1}$ by $R(\lambda, A)$, where $I$ is the identity operator. If $A \in \mathbf{B}(X)$ is quasi-nilpotent, then for any complex $\lambda \neq 0$

$$
R(\lambda, A)=\sum_{k=0}^{\infty} \lambda^{-k-1} A^{k} .
$$

For a deeper discussion of the theory of operator, we refer the reader to [32].

If $A$ is generalized Drazin invertible, then the spectral idempotent $A^{\pi}$ of $A$ corresponding to $\{0\}$ is given by $A^{\pi}=I-A A^{d}$.

Lemma 2.1. If $A$ and $D$ are quasi-nilpotent and $B D^{i} C=0$, for any nonnegative integer $i$, then $M$ is quasi-nilpotent.

Proof. From (2.2) we can verify that $B R(\lambda, D) C=0$ for any complex $\lambda \neq 0$. Since $A$ and $D$ are quasi-nilpotent, it follows that

$$
R(\lambda, M)=\left(\begin{array}{cc}
R(\lambda, A) & R(\lambda, A) B R(\lambda, D) \\
R(\lambda, D) C R(\lambda, A) & R(\lambda, D)+R(\lambda, D) C R(\lambda, A) B R(\lambda, D)
\end{array}\right)
$$

for any complex $\lambda \neq 0$. Thus $\sigma(M) \subseteq \sigma(A) \cup \sigma(D)=\{0\}$, implying that $M$ is quasinilpotent. 
Lemma 2.2 ([17]). If $P, Q \in \mathbf{B}(X)$ are generalized Drazin invertible and $P Q=0$, then $P+Q$ is generalized Drazin invertible and

$$
(P+Q)^{d}=Q^{\pi} \sum_{i=0}^{\infty} Q^{i}\left(P^{d}\right)^{i+1}+\sum_{i=0}^{\infty}\left(Q^{d}\right)^{i+1} P^{i} P^{\pi} .
$$

Lemma 2.3 ([1]). For $B \in \mathbf{B}(X, Y)$ and $C \in \mathbf{B}(Y, X), B C$ is generalized Drazin invertible if and only if $C B$ is generalized Drazin invertible. In this case, $\left((B C)^{d}\right)^{i}=B\left((C B)^{d}\right)^{i+1} C$, for any positive integer $i$.

For notational convenience, we define a sum to be 0 , whenever its lower limit is bigger than its upper limit. We define $A^{0}=I$.

\section{Main results}

We start with a special case of our main results, which is of independent interest.

Lemma 3.1. If $A$ is generalized Drazin invertible, $D$ is quasi-nilpotent and $B D^{i} C=0$, for any nonnegative integer $i$, then $M$ is generalized Drazin invertible and

$$
M^{d}=\left(\begin{array}{cc}
A^{d} & \Gamma \\
\Delta & \Delta A \Gamma
\end{array}\right)
$$

where $\Gamma=\sum_{i=0}^{\infty}\left(A^{d}\right)^{i+2} B D^{i}$ and $\Delta=\sum_{i=0}^{\infty} D^{i} C\left(A^{d}\right)^{i+2}$.

Proof. It is easy to check that $\Gamma D^{i} C=0, B D^{i} \Delta=0$ and $\Gamma D^{i} \Delta=0$, for any nonnegative integer $i$. Let $W$ be defined as in (3.1). We first prove that $M W=W M$. Since $B \Delta=0$ and $\Gamma C=0$, it follows that

$$
\begin{aligned}
M W & =\left(\begin{array}{cc}
A A^{d} & A \Gamma \\
C A^{d}+D \Delta & C \Gamma+D \Delta A \Gamma
\end{array}\right), \\
W M & =\left(\begin{array}{cc}
A^{d} A & A^{d} B+\Gamma D \\
\Delta A & \Delta B+\Delta A \Gamma D
\end{array}\right) .
\end{aligned}
$$

We can verify that

$$
\begin{aligned}
& A^{d} B+\Gamma D=A^{d} B+\sum_{i=0}^{\infty}\left(A^{d}\right)^{i+2} B D^{i+1}=\sum_{i=0}^{\infty}\left(A^{d}\right)^{i+1} B D^{i}=A \Gamma, \\
& C A^{d}+D \Delta=C A^{d}+\sum_{i=0}^{\infty} D^{i+1} C\left(A^{d}\right)^{i+2}=\sum_{i=0}^{\infty} D^{i} C\left(A^{d}\right)^{i+1}=\Delta A .
\end{aligned}
$$

Since $A A^{d} \Gamma=\Gamma$ and $\Delta A A^{d}=\Delta$, the equation (3.2) yields

$$
\begin{aligned}
C \Gamma+D \Delta A \Gamma & =C \Gamma+\left(\Delta A-C A^{d}\right) A \Gamma=C \Gamma+\Delta A^{2} \Gamma-C \Gamma \\
& =\Delta A^{2} \Gamma=\Delta A\left(A^{d} B+\Gamma D\right)=\Delta B+\Delta A \Gamma D .
\end{aligned}
$$

Thus $M W=W M$.

Next, we will prove that $W=W^{2} M$. Since $\Gamma \Delta=0$ and $\Delta A A^{d}=\Delta$, we get

$$
W^{2} M=\left(\begin{array}{cc}
A^{d} & \left(A^{d}\right)^{2} B+A^{d} \Gamma D \\
\Delta & \Delta A^{d} B+\Delta \Gamma D
\end{array}\right)
$$

Since $A \Gamma=A^{d} B+\Gamma D$ by (3.2), we have

$$
\begin{aligned}
\left(A^{d}\right)^{2} B+A^{d} \Gamma D & =A A^{d} \Gamma=\Gamma, \\
\Delta A^{d} B+\Delta \Gamma D & =\Delta A \Gamma .
\end{aligned}
$$

Thus $W=W^{2} M$. 
Finally, we will prove that $M-M^{2} W$ is quasi-nilpotent. Since $B D^{i} C=0$ and $B D^{i} \Delta=$ 0 , for any nonnegative integer $i$, a calculation yields

$$
M-M^{2} W=\left(\begin{array}{cc}
A A^{\pi} & B-A^{2} \Gamma \\
C A^{\pi}-D C A^{d}-D^{2} \Delta & D-\Sigma
\end{array}\right),
$$

where $\Sigma=C A \Gamma+D C \Gamma+D^{2} \Delta A \Gamma$. From $\Gamma D^{i} C=0$ and $\Gamma D^{i} \Delta=0$, it follows that $\Sigma D^{i} \Sigma=$ 0 for any integer $i \geq 0$. Since $D$ is quasi-nilpotent, by $(2.2)$ we have $\Sigma R(\lambda, D) \Sigma=0$ for any $\lambda \neq 0$, whence

$$
(\lambda I-D+\Sigma)(R(\lambda, D)-R(\lambda, D) \Sigma R(\lambda, D))=I .
$$

Hence $R(\lambda, D-\Sigma)=R(\lambda, D)-R(\lambda, D) \Sigma R(\lambda, D)$ for any $\lambda \neq 0$, which implies that $D-\Sigma$ is quasi-nilpotent. By Lemma 2.1, $M-M^{2} W$ is quasi-nilpotent. Thus $W$ is the generalized Drazin inverse of $M$.

We are now in a position to prove our main results.

Theorem 3.2. Let $M$ be defined as in (2.1) such that $A$ and $D$ are generalized Drazin invertible. If $B D^{d}=0$ and $B D^{i} C=0$, for any nonnegative integer $i$, then $M$ is generalized Drazin invertible and

where

$$
M^{d}=\left(\begin{array}{cc}
A^{d} & \Gamma \\
\Sigma_{0} & D^{d}+\Lambda
\end{array}\right)
$$

$$
\begin{aligned}
\Gamma= & \sum_{i=0}^{\infty}\left(A^{d}\right)^{i+2} B D^{i}, \\
\Sigma_{0}= & D^{\pi} \sum_{i=0}^{\infty} D^{i} C\left(A^{d}\right)^{i+2}+\sum_{i=0}^{\infty}\left(D^{d}\right)^{i+2} C A^{i} A^{\pi}-D^{d} C A^{d}, \\
\Lambda= & D^{\pi} \sum_{i=0}^{\infty} \sum_{j=0}^{\infty} D^{i} C\left(A^{d}\right)^{i+j+3} B D^{j}+\sum_{i=0}^{\infty} \sum_{j=0}^{i}\left(D^{d}\right)^{i+3} C A^{j} B D^{i-j} \\
& -\sum_{i=0}^{\infty} \sum_{j=0}^{\infty}\left(D^{d}\right)^{i+1} C A^{i}\left(A^{d}\right)^{j+2} B D^{j} .
\end{aligned}
$$

Proof. Let $P=\left(\begin{array}{cc}A & B \\ C & D D^{\pi}\end{array}\right)$ and $Q=\left(\begin{array}{cc}0 & 0 \\ 0 & D^{2} D^{d}\end{array}\right)$. Then $M=P+Q$, and $P Q=0$. By [27, Theorem 5.4], $\left(D^{2} D^{d}\right)^{d}=\left(\left(D^{d}\right)^{d}\right)^{d}=D^{d}$. Hence

$$
Q^{d}=\left(\begin{array}{cc}
0 & 0 \\
0 & D^{d}
\end{array}\right) \text { and } Q^{\pi}=\left(\begin{array}{cc}
I & 0 \\
0 & D^{\pi}
\end{array}\right)
$$

and so $Q Q^{\pi}=0$. It follows from Lemma 2.2 that

$$
M^{d}=Q^{\pi} P^{d}+\sum_{i=0}^{\infty}\left(Q^{d}\right)^{i+1} P^{\pi} P^{i}
$$

Note that $D D^{\pi}$ is quasi-nilpotent and $B\left(D D^{\pi}\right)^{i} C=B D^{i} C=0$, for any nonnegative integer $i$, since $B D^{\pi}=B$. We can apply Lemma 3.1 to $P$ with $D$ replaced by $D D^{\pi}$, to obtain $P^{d}=\left(\begin{array}{cc}A^{d} & \Gamma \\ \Delta^{\prime} & \Delta^{\prime} A \Gamma\end{array}\right)$, where $\Delta^{\prime}=\sum_{i=0}^{\infty}\left(D D^{\pi}\right)^{i} C\left(A^{d}\right)^{i+2}$. Hence $D^{\pi} \Delta^{\prime}=D^{\pi} \Delta$ and

$$
Q^{\pi} P^{d}=\left(\begin{array}{cc}
A^{d} & \Gamma \\
D^{\pi} \Delta & D^{\pi} \Delta A \Gamma
\end{array}\right) .
$$

Note that $B \Delta^{\prime}=0$. A calculation yields

$$
P^{\pi}=I-P P^{d}=\left(\begin{array}{cc}
A^{\pi} & -A \Gamma \\
-C A^{d}-D D^{\pi} \Delta & I-C \Gamma-D D^{\pi} \Delta A \Gamma
\end{array}\right) .
$$


Since $B\left(D D^{\pi}\right)^{i} C=0$, for any positive integer $i$, by induction on $i \geq 1$ we deduce that $P^{i}=\left(\begin{array}{cc}A^{i} & B_{i} \\ C_{i} & D^{i} D^{\pi}+N_{i}\end{array}\right)$, where

$$
\begin{aligned}
B_{i} & =\sum_{m=0}^{i-1} A^{m} B D^{i-1-m}, \\
C_{i} & =\sum_{m=0}^{i-1}\left(D D^{\pi}\right)^{m} C A^{i-1-m}, \\
N_{i} & =\sum_{m=0}^{i-2}\left(D D^{\pi}\right)^{m} C \sum_{n=0}^{i-2-m} A^{n} B D^{i-2-m-n} .
\end{aligned}
$$

Now we can check that

$$
\begin{aligned}
\sum_{i=1}^{\infty}\left(Q^{d}\right)^{i+1} P^{i} & =\sum_{i=1}^{\infty}\left(\begin{array}{cc}
0 & 0 \\
0 & \left(D^{d}\right)^{i+1}
\end{array}\right)\left(\begin{array}{cc}
A^{i} & B_{i} \\
C_{i} & D^{i} D^{i}+N_{i}
\end{array}\right) \\
& =\sum_{i=1}^{\infty}\left(\begin{array}{cc}
0 & 0 \\
\left(D^{d}\right)^{i+1} C A^{i-1} & \left(D^{d}\right)^{i+1} C \sum_{n=0}^{i-2} A^{n} B D^{i-2-n}
\end{array}\right) \\
& =\left(\begin{array}{cc}
0 & 0 \\
\sum_{i=0}^{\infty}\left(D^{d}\right)^{i+2} C A^{i} & \sum_{i=2}^{\infty}\left(D^{d}\right)^{i+1} C \sum_{n=0}^{i-2} A^{n} B D^{i-2-n}
\end{array}\right)
\end{aligned}
$$

Since $B D^{i} C=0, B D^{d}=0$ and $B D^{i} \Delta=0$, we have

$$
\begin{aligned}
& \sum_{i=1}^{\infty}\left(Q^{d}\right)^{i+1} P^{i} P^{\pi}= \\
& \left(\begin{array}{cc}
0 & 0 \\
\sum_{i=0}^{\infty}\left(D^{d}\right)^{i+2} C A^{i} A^{\pi} & \sum_{i=0}^{\infty}\left(D^{d}\right)^{i+3} C \sum_{n=0}^{i} A^{n} B D^{i-n}-\sum_{i=0}^{\infty}\left(D^{d}\right)^{i+2} C A^{i+1} \Gamma
\end{array}\right) .
\end{aligned}
$$

By $Q^{d} P^{\pi}=\left(\begin{array}{cc}0 & 0 \\ -D^{d} C A^{d} & D^{d}-D^{d} C \Gamma\end{array}\right)$, we have

$$
\sum_{i=0}^{\infty}\left(Q^{d}\right)^{i+1} P^{i} P^{\pi}=\left(\begin{array}{cc}
0 & 0 \\
\sum_{i=0}^{\infty}\left(D^{d}\right)^{i+2} C A^{i} A^{\pi}-D^{d} C A^{d} & D^{d}+\Omega
\end{array}\right),
$$

where

$$
\Omega=\sum_{i=0}^{\infty}\left(D^{d}\right)^{i+3} C \sum_{j=0}^{i} A^{j} B D^{i-j}-\sum_{i=0}^{\infty}\left(D^{d}\right)^{i+1} C A^{i} \Gamma .
$$

Combining (3.5) and (3.6) with (3.4) gives

$$
\begin{aligned}
M^{d} & =\left(\begin{array}{cc}
A^{d} & \Gamma \\
D^{\pi} \Delta+\sum_{i=0}^{\infty}\left(D^{d}\right)^{i+2} C A^{i} A^{\pi}-D^{d} C A^{d} & D^{d}+D^{\pi} \Delta A \Gamma+\Omega
\end{array}\right) \\
& =\left(\begin{array}{cc}
A^{d} & \Gamma \\
\Sigma_{0} & D^{d}+\Lambda
\end{array}\right) .
\end{aligned}
$$

Let $A^{*}$ denote the conjugate operator of an operator $A$. Then $\left(A^{d}\right)^{*}=\left(A^{*}\right)^{d}$ by [28, Lemma 1.3]. Let $M^{*}=\left(\begin{array}{cc}A^{*} & C^{*} \\ B^{*} & D^{*}\end{array}\right)=\left(\begin{array}{cc}A_{1} & B_{1} \\ C_{1} & D_{1}\end{array}\right)$, then $B_{1} D_{1}^{i} C_{1}=C^{*}\left(D^{*}\right)^{i} B^{*}=$ $\left(B D^{i} C\right)^{*}$ and $B_{1} D_{1}^{d}=C^{*}\left(D^{*}\right)^{d}=\left(D^{d} C\right)^{*}$. Applying Theorem 3.2 to $M^{*}=\left(\begin{array}{cc}A^{*} & C^{*} \\ B^{*} & D^{*}\end{array}\right)$ gives the representation for the generalized Drazin inverse of $M$ satisfying the following condition. 
Corollary 3.1. If $A$ and $D$ are generalized Drazin invertible and $D^{d} C=0$ and $B D^{i} C=0$, for any nonnegative integer $i$, then $M$ is generalized Drazin invertible and

$$
M^{d}=\left(\begin{array}{cc}
A^{d} & S \\
\Gamma_{1} & D^{d}+\Lambda_{1}
\end{array}\right),
$$

where

$$
\begin{aligned}
\Gamma_{1}= & \sum_{i=0}^{\infty} D^{i} C\left(A^{d}\right)^{i+2}, \\
S= & A^{\pi} \sum_{i=0}^{\infty} A^{i} B\left(D^{d}\right)^{i+2}+\sum_{i=0}^{\infty}\left(A^{d}\right)^{i+2} B D^{i} D^{\pi}-A^{d} B D^{d}, \\
\Lambda_{1}= & \sum_{i=0}^{\infty} \sum_{j=0}^{\infty} D^{i} C\left(A^{d}\right)^{i+j+3} B D^{j} D^{\pi}+\sum_{i=0}^{\infty} \sum_{j=0}^{i} D^{i-j} C A^{j} B\left(D^{d}\right)^{i+3} \\
& -\sum_{i=0}^{\infty} \sum_{j=0}^{\infty} D^{i} C\left(A^{d}\right)^{i+2} A^{j} B\left(D^{d}\right)^{j+1} .
\end{aligned}
$$

Furthermore, the mapping $M \mapsto \bar{M}=\left(\begin{array}{cc}D & C \\ B & A\end{array}\right)$ is an isometric isomorphism from $B(X \oplus Y)$ to $B(Y \oplus X)$ and $\bar{M}^{*}=\overline{M^{*}}$. Applying the theorem 3.2 to $\left(\begin{array}{cc}D & C \\ B & A\end{array}\right)$ and $\left(\begin{array}{ll}D^{*} & B^{*} \\ C^{*} & A^{*}\end{array}\right)$ respectively, gives the following two corollaries. The following corollary generalizes $[13$, Theorem $6(3)]$.

Corollary 3.2. If $A$ and $D$ are generalized Drazin invertible and $C A^{d}=0$ and $C A^{i} B=0$, for any nonnegative integer $i$, then $M$ is generalized Drazin invertible and

$$
M^{D}=\left(\begin{array}{cc}
A^{d}+Z & S \\
\Psi & D^{d}
\end{array}\right),
$$

where

$$
\begin{aligned}
\Psi= & \sum_{i=0}^{\infty}\left(D^{d}\right)^{i+2} C A^{i}, \\
S= & A^{\pi} \sum_{i=0}^{\infty} A^{i} B\left(D^{d}\right)^{i+2}+\sum_{i=0}^{\infty}\left(A^{d}\right)^{i+2} B D^{i} D^{\pi}-A^{d} B D^{d}, \\
Z= & A^{\pi} \sum_{i=0}^{\infty} \sum_{j=0}^{\infty} A^{i} B\left(D^{d}\right)^{i+j+3} C A^{j}-\sum_{i=0}^{\infty} \sum_{j=0}^{\infty}\left(A^{d}\right)^{i+1} B D^{i}\left(D^{d}\right)^{j+2} C A^{j} \\
& +\sum_{i=0}^{\infty} \sum_{j=0}^{i}\left(A^{d}\right)^{i+3} B D^{j} C A^{i-j} .
\end{aligned}
$$

Corollary 3.3. If $A$ and $D$ are generalized Drazin invertible and $A^{d} B=0$ and $C A^{i} B=0$, for any nonnegative integer $i$, then $M$ is generalized Drazin invertible and

$$
M^{D}=\left(\begin{array}{cc}
A^{d}+Z_{1} & \widetilde{\Psi} \\
\Sigma_{0} & D^{d}
\end{array}\right),
$$


where $\Sigma_{0}$ is as in (3.3) and

$$
\begin{aligned}
\widetilde{\Psi} & =\sum_{i=0}^{\infty} A^{i} B\left(D^{d}\right)^{i+2} \\
Z_{1}= & \sum_{i=0}^{\infty} \sum_{j=0}^{\infty} A^{i} B\left(D^{d}\right)^{i+j+3} C A^{j} A^{\pi}+\sum_{i=0}^{\infty} \sum_{j=0}^{i} A^{i-j} B D^{j} C\left(A^{d}\right)^{i+3} \\
& \quad-\sum_{i=0}^{\infty} \sum_{j=0}^{\infty} A^{i} B\left(D^{d}\right)^{i+2} D^{j} C\left(A^{d}\right)^{j+1}
\end{aligned}
$$

The following result is a direct corollary of Corollary 3.3, the conditions of which were considered in [9, Theorem 2.10].

Corollary 3.4. If $A$ and $D$ are generalized Drazin invertible and $A A^{d} B=0$ and $C(I-$ $\left.A A^{d}\right)=0$, then $M$ is generalized Drazin invertible and

$$
M^{D}=\left(\begin{array}{cc}
A^{d}+Z^{\prime} & \Psi^{\prime} \\
\Sigma_{0} & D^{d}
\end{array}\right),
$$

where

$$
\begin{aligned}
& \Psi^{\prime}=\sum_{i=0}^{\infty} A^{i} B\left(D^{d}\right)^{i+2}, \\
& Z^{\prime}=\sum_{i=0}^{\infty} \sum_{j=0}^{i} A^{i-j} B D^{j} C\left(A^{d}\right)^{i+3}-\sum_{i=0}^{\infty} \sum_{j=0}^{\infty} A^{i} B\left(D^{d}\right)^{i+2} D^{j} C\left(A^{d}\right)^{j+1} .
\end{aligned}
$$

Proof. Since $A A^{d} B=0$ and $C\left(I-A A^{d}\right)=0$, we have $A^{d} B=0$ and $C A^{i} B=C A^{i}(I-$ $\left.A A^{d}\right) B=0$, for any nonnegative integer $i$. So $M$ satisfies the condition of Corollary 3.3 .

The following result is a direct corollary of Theorem 3.2, which extends [18, Theorem $2.2]$ to bounded linear operators on a Banach space, and generalizes the results in $[9,13,16]$.

Corollary 3.5. If $A$ and $D$ are generalized Drazin invertible and $B C=0, B D C=0$ and $B D^{2}=0$, then $M$ is generalized Drazin invertible and

$$
M^{d}=\left(\begin{array}{cc}
A^{d} & \left(A^{d}\right)^{3}(A B+B D) \\
\Sigma_{0} & D^{d}+\left(D^{d}\right)^{3} C B+\Sigma_{2}(A B+B D)
\end{array}\right)
$$

where

$$
\Sigma_{n}=\sum_{i=0}^{\infty}\left(D^{d}\right)^{i+n+2} C A^{i} A^{\pi}+D^{\pi} \sum_{i=0}^{\infty} D^{i} C\left(A^{d}\right)^{i+n+2}-\sum_{i=0}^{n}\left(D^{d}\right)^{i+1} C\left(A^{d}\right)^{n-i+1} .
$$

Proof. It is sufficient to simplify $\Gamma$ and $\Lambda$ in Theorem 3.2 to the form given here under the assumption that $B C=0, B D C=0$ and $B D^{2}=0$. Clearly $\Gamma=\left(A^{d}\right)^{3}(A B+B D)$. We can check that

$$
\begin{aligned}
\Lambda= & D^{\pi} \sum_{i=0}^{\infty} D^{i} C\left(A^{d}\right)^{i+4}(A B+B D)-\sum_{i=0}^{\infty}\left(D^{d}\right)^{i+1} C A^{i}\left(A^{d}\right)^{3}(A B+B D) \\
& +\sum_{i=0}^{\infty}\left(D^{d}\right)^{i+3} C A^{i} B+\sum_{i=1}^{\infty}\left(D^{d}\right)^{i+3} C A^{i-1} B D
\end{aligned}
$$




$$
\begin{aligned}
= & D^{\pi} \sum_{i=0}^{\infty} D^{i} C\left(A^{d}\right)^{i+4}(A B+B D)-\sum_{i=0}^{2}\left(D^{d}\right)^{i+1} C\left(A^{d}\right)^{3-i}(A B+B D) \\
& -\sum_{i=3}^{\infty}\left(D^{d}\right)^{i+1} C A^{i-3} A^{3}\left(A^{d}\right)^{3}(A B+B D)+\left(D^{d}\right)^{3} C B \\
& +\sum_{i=1}^{\infty}\left(D^{d}\right)^{i+3} C A^{i} B+\sum_{i=1}^{\infty}\left(D^{d}\right)^{i+3} C A^{i-1} B D \\
= & D^{\pi} \sum_{i=0}^{\infty} D^{i} C\left(A^{d}\right)^{i+4}(A B+B D)-\sum_{i=0}^{2}\left(D^{d}\right)^{i+1} C\left(A^{d}\right)^{3-i}(A B+B D) \\
& +\sum_{i=0}^{\infty}\left(D^{d}\right)^{i+4} C A^{i} A^{\pi}(A B+B D)+\left(D^{d}\right)^{3} C B \\
= & \left(D^{d}\right)^{3} C B+\Sigma_{2}(A B+B D) .
\end{aligned}
$$

The following result is a corollary of Theorem 3.2, the conditions of which are considered in [18, Theorem 2.5] for matrices.

Corollary 3.6. If $A$ and $D$ are generalized Drazin invertible and $B D^{\pi} C=0, B D^{d}=0$ and $D D^{\pi} C=0$, then $M$ is generalized Drazin invertible and

$$
M^{d}=\left(\begin{array}{cc}
A^{d} & \Gamma \\
D^{\pi} C\left(A^{d}\right)^{2}+\sum_{i=0}^{\infty}\left(D^{d}\right)^{i+2} C A^{i} A^{\pi}-D^{d} C A^{d} & D^{d}+E
\end{array}\right),
$$

where $\Gamma$ is as in (3.3) and

$$
E=D^{\pi} C A^{d} \Gamma+\sum_{i=0}^{\infty} \sum_{j=0}^{i}\left(D^{d}\right)^{i+3} C A^{j} B D^{i-j}-\sum_{i=0}^{\infty}\left(D^{d}\right)^{i+1} C A^{i} \Gamma .
$$

Proof. It is sufficient to check that $M$ satisfies the condition of Theorem 3.2. Since $B D^{d}=0$, we have $B D^{d} D C=0$. Hence $B D^{\pi} C=0$ implies $B C=0$, and $D D^{\pi} C=0$ implies $D C=D^{d} D^{2} C$. Thus $B D^{i} C=B D^{d} D^{i+1} C=0$, for any nonnegative integer $i$.

\section{Applications}

In this section, we first derive some representations for the generalized Drazin inverse of $M$ with application of Theorem 3.2.

Theorem 4.1. Let $M$ be defined as in (2.1) such that $A$ and $D$ are generalized Drazin invertible. If

$$
B D^{d}=0, \quad D^{\pi} C A=0 \text { and } D^{\pi} C B=0,
$$

then $M$ is generalized Drazin invertible and

$$
M^{d}=\left(\begin{array}{cc}
A^{d}+A^{d} \Gamma C & \Gamma \\
T-D^{d} C A^{d} \Gamma C+D^{d} \Lambda^{\prime} C & D^{d}+\Lambda^{\prime}
\end{array}\right),
$$

where $\Gamma$ is as in (3.3) and

$$
\begin{aligned}
T & =\sum_{i=0}^{\infty}\left(D^{d}\right)^{i+2} C A^{i} A^{\pi}-D^{d} C A^{d}, \\
\Lambda^{\prime} & =\sum_{i=0}^{\infty} \sum_{j=0}^{i}\left(D^{d}\right)^{i+3} C A^{j} B D^{i-j}-\sum_{i=0}^{\infty} \sum_{j=0}^{\infty}\left(D^{d}\right)^{i+1} C A^{i}\left(A^{d}\right)^{j+2} B D^{j} .
\end{aligned}
$$


Proof. Let

$$
P=\left(\begin{array}{cc}
0 & 0 \\
D^{\pi} C & 0
\end{array}\right) \text { and } Q=\left(\begin{array}{cc}
A & B \\
D D^{d} C & D
\end{array}\right) .
$$

Then $M=P+Q, P Q=0$, and $P^{2}=0$. Hence Lemma 2.2 implies that

$$
M^{d}=Q^{d}+\left(Q^{d}\right)^{2} P .
$$

Since $B D^{d}=0$ and $B D^{i}\left(D D^{d} C\right)=0$, for any nonnegative integer $i$, we can apply Theorem 3.2 to $Q$ to obtain

$$
Q^{d}=\left(\begin{array}{cc}
A^{d} & \Gamma \\
T & D^{d}+\Lambda^{\prime}
\end{array}\right) .
$$

Note that $\Gamma D^{d}=0, \Gamma \Lambda^{\prime}=0, \Lambda^{\prime} D^{d}=0, \Lambda^{\prime 2}=0, \Gamma D^{\pi}=\Gamma$ and $\Lambda^{\prime} D^{\pi}=\Lambda^{\prime}$. We can check that

$$
\left(Q^{d}\right)^{2} P=\left(\begin{array}{cc}
* & A^{d} \Gamma \\
* & \left(D^{d}\right)^{2}+T \Gamma+D^{d} \Lambda^{\prime}
\end{array}\right) P=\left(\begin{array}{cc}
A^{d} \Gamma C & 0 \\
T \Gamma C+D^{d} \Lambda^{\prime} C & 0
\end{array}\right),
$$

where $*$ denotes entries we need not specify, $\Gamma$ is as in Lemma 3.1 and $T, \Lambda^{\prime}$ are as in (4.2). Since $T \Gamma=-D^{d} C A^{d} \Gamma$, we conclude that

$$
M^{d}=\left(\begin{array}{cc}
A^{d}+A^{d} \Gamma C & \Gamma \\
T-D^{d} C A^{d} \Gamma C+D^{d} \Lambda^{\prime} C & D^{d}+\Lambda^{\prime}
\end{array}\right) .
$$

As a special case of Theorem 4.1, the following corollary extends [18, Theorem 2.7] to bounded linear operators on a Banach space.

Corollary 4.1. If $A$ and $D$ are generalized Drazin invertible and

$$
B D=0, \quad D^{\pi} C A=0 \text { and } D^{\pi} C B=0,
$$

then $M$ is generalized Drazin invertible and

$$
\left(\begin{array}{cc}
A^{d}+\left(A^{d}\right)^{3} B C & \left(A^{d}\right)^{2} B \\
\Upsilon_{0}+\Upsilon_{2} B C & D^{D}+\Upsilon_{1} B
\end{array}\right)
$$

where

$$
\Upsilon_{n}=\sum_{i=0}^{\infty}\left(D^{d}\right)^{i+n+2} C A^{i} A^{\pi}-\sum_{i=0}^{n}\left(D^{d}\right)^{i+1} C\left(A^{d}\right)^{n-i+1}, n=0,1,2 .
$$

The rest of this section is devoted to a generalization of Theorem 3.2 by changing the condition $B C=0$ to $A B C=0$. We start with the following additive result.

Theorem 4.2. If $P, Q \in \mathbf{B}(X)$ are generalized Drazin invertible, $P Q^{d}=0$ and $P Q^{i} P=0$, for any integer $i \geq 1$, then $P+Q$ is generalized Drazin invertible and

$$
\begin{aligned}
(P+Q)^{d}= & Q^{\pi} \sum_{i=0}^{\infty} \sum_{j=0}^{\infty} Q^{i}\left(P^{d}\right)^{i+j+1} Q^{j}+\sum_{i=0}^{\infty}\left(Q^{d}\right)^{i+1} P^{i} P^{\pi} \\
& -\sum_{i=0}^{\infty} \sum_{j=0}^{\infty}\left(Q^{d}\right)^{i+1} P^{i}\left(P^{d}\right)^{j+1} Q^{j+1}+\sum_{i=0}^{\infty} \sum_{j=0}^{i}\left(Q^{d}\right)^{i+3} P^{j+1} Q^{i-j+1} .
\end{aligned}
$$

Proof. Let $Y=\overline{R(P)}$. Let $B: X \rightarrow Y$ and $C: Y \rightarrow X$ be defined by $B(x)=P(x)$ and $C(y)=y, x \in X, y \in Y$. Evidenty, $B, C$ are linear bounded operators and $P=C B$. By $P Q^{d}=0$, we have $C B Q^{d}=0$. Because $C$ is a inclusion mapping, we have $B Q^{d}=0$. By $P Q^{i} P=0$, we have $C B Q^{i} C B=0$ and then $B Q^{i} C B=0$. 
Note that $R(B)=R(P)$ is dense in $Y$ and $B Q^{i} C$ are bounded linear operators, so we have $B Q^{i} C=0$, for any integer $i \geq 1$. By Lemma 2.3, we obtain that

$$
(P+Q)^{d}=\left((C I)\left(\begin{array}{c}
B \\
Q
\end{array}\right)\right)^{d}=(C I)\left(\left(\begin{array}{cc}
B C & B \\
Q C & Q
\end{array}\right)^{d}\right)^{2}\left(\begin{array}{c}
B \\
Q
\end{array}\right) .
$$

Since $B Q^{d}=0$ and $B Q^{i} C=0$ for $i \geq 1$, Theorem 3.2 shows that

$$
\left(\begin{array}{cc}
B C & B \\
Q C & Q
\end{array}\right)^{d}=\left(\begin{array}{cc}
(B C)^{d} & \Gamma^{\prime} \\
\Sigma_{0}^{\prime} & Q^{d}+\Lambda^{\prime \prime}
\end{array}\right)
$$

where

$$
\begin{aligned}
\Gamma^{\prime} & =\sum_{i=0}^{\infty}\left((B C)^{d}\right)^{i+2} B Q^{i}, \\
\Sigma_{0}^{\prime} & =Q^{\pi} \sum_{i=0}^{\infty} Q^{i+1} C\left((B C)^{d}\right)^{i+2}+\sum_{i=0}^{\infty}\left(Q^{d}\right)^{i+1} C(B C)^{i}(B C)^{\pi}-Q Q^{d} C(B C)^{d}, \\
\Lambda^{\prime \prime} & =Q^{\pi} \sum_{i=0}^{\infty} \sum_{j=0}^{\infty} Q^{i+1} C\left((B C)^{d}\right)^{i+j+3} B Q^{j}+\sum_{i=0}^{\infty} \sum_{j=0}^{i}\left(Q^{d}\right)^{i+2} C(B C)^{j} B Q^{i-j} \\
& -\sum_{i=0}^{\infty}\left(Q^{d}\right)^{i+1} Q C(B C)^{i} \Gamma^{\prime} .
\end{aligned}
$$

Since $\Gamma^{\prime} \Sigma_{0}^{\prime}=0, \Gamma^{\prime} Q^{d}=0, \Gamma^{\prime} \Lambda^{\prime \prime}=0, \Lambda^{\prime \prime} \Sigma_{0}^{\prime}=0, \Lambda^{\prime \prime} Q^{d}=0$ and $\left(\Lambda^{\prime \prime}\right)^{2}=0$, therefore

$$
\left(\left(\begin{array}{cc}
B C & B \\
Q C & Q
\end{array}\right)^{d}\right)^{2}=\left(\begin{array}{cc}
\left((B C)^{d}\right)^{2} & (B C)^{d} \Gamma^{\prime} \\
\Sigma_{0}^{\prime}(B C)^{d}+Q^{d} \Sigma_{0}^{\prime} & \Sigma_{0}^{\prime} \Gamma^{\prime}+\left(Q^{d}\right)^{2}+Q^{d} \Lambda^{\prime \prime}
\end{array}\right) .
$$

Substitute the equation above into (4.6), we obtain

$$
\begin{aligned}
(P+Q)^{d}= & C\left((B C)^{d}\right)^{2} B+Q^{d}+\Sigma_{0}^{\prime}(B C)^{d} B+Q^{d} \Sigma_{0}^{\prime} B \\
& +C(B C)^{d} \Gamma^{\prime} Q+\Sigma_{0}^{\prime} \Gamma^{\prime} Q+Q^{d} \Lambda^{\prime \prime} Q \\
= & (C B)^{d}+Q^{d}+\Sigma_{0}^{\prime}(B C)^{d} B+Q^{d} \Sigma_{0}^{\prime} B \\
& +C(B C)^{d} \Gamma^{\prime} Q+\Sigma_{0}^{\prime} \Gamma^{\prime} Q-\sum_{i=0}^{\infty}\left(Q^{d}\right)^{i+1} C(B C)^{i} \Gamma^{\prime} Q \\
& +\sum_{i=0}^{\infty} \sum_{j=0}^{i}\left(Q^{d}\right)^{i+3}(C B)^{j+1} Q^{i-j+1} .
\end{aligned}
$$

We can check that

$$
\begin{aligned}
& (C B)^{d}+\Sigma_{0}^{\prime}(B C)^{d} B=Q^{\pi} \sum_{i=0}^{\infty} Q^{i}\left((C B)^{d}\right)^{i+1}, \\
& Q^{d}+Q^{d} \Sigma_{0}^{\prime} B=\sum_{i=0}^{\infty}\left(Q^{d}\right)^{i+1}(C B)^{i}(C B)^{\pi},
\end{aligned}
$$

and

$$
\begin{aligned}
C(B C)^{d}+\Sigma_{0}^{\prime}-\sum_{i=0}^{\infty}\left(Q^{d}\right)^{i+1} C(B C)^{i} \\
=Q^{\pi} \sum_{i=0}^{\infty} Q^{i} C\left((B C)^{d}\right)^{i+1}-\sum_{i=0}^{\infty}\left(Q^{d}\right)^{i+1} C(B C)^{i+1}(B C)^{d} \\
=Q^{\pi} \sum_{i=0}^{\infty} Q^{i}\left((C B)^{d}\right)^{i+1} C-\sum_{i=0}^{\infty}\left(Q^{d}\right)^{i+1}(C B)^{i+1}(C B)^{d} C
\end{aligned}
$$


Substituting (4.8) and (4.10) into (4.7) and noting that $C \Gamma^{\prime} Q=\sum_{i=0}^{\infty}\left((C B)^{d}\right)^{i+1} Q^{i+1}$, we can get the desired expression of $(P+Q)^{d}$.

As corollary of Theorem 4.2, the following result extends the main result in [34] to bounded linear operators on a Banach space.

Corollary 4.2. If $P, Q \in \mathbf{B}(X)$ are generalized Drazin invertible, $P Q P=0$ and $P Q^{2}=0$, then $P+Q$ is generalized Drazin invertible and

$$
\begin{aligned}
(P+Q)^{d}=Q^{\pi} \sum_{i=0}^{\infty} Q^{i}\left(P^{d}\right)^{i+1}+Q^{\pi} \sum_{i=0}^{\infty} Q^{i}\left(P^{d}\right)^{i+2} Q+\sum_{i=0}^{\infty}\left(Q^{d}\right)^{i+1} P^{i} P^{\pi} & \\
& +\sum_{i=0}^{\infty}\left(Q^{d}\right)^{i+3} P^{i+1} P^{\pi} Q-Q^{d} P^{d} Q-\left(Q^{d}\right)^{2} P P^{d} Q .
\end{aligned}
$$

Now, we give another result. In this case, the representations are quite complex.

Theorem 4.3. Let $M$ be the form defined by (2.1) such that $A, D$ and $B C$ are generalized Drazin invertible. If

$$
B D^{d}=0, A B C=0 \text { and } B D^{i} C=0,
$$

for any positive integer $i$, then $M$ is generalized Drazin invertible and

$$
M^{d}=\left(\begin{array}{cc}
\Phi_{1} A & \Phi_{1} B+\sum_{i=0}^{\infty} \Phi_{i+2}(A B+B D) D^{2 i+1} \\
\widetilde{\Sigma}_{0} A+\Psi_{1} & \widetilde{\Sigma}_{0} B+\left(C B+D^{2}\right)^{d} D+\widetilde{\Lambda} D
\end{array}\right),
$$

where

$$
\begin{gathered}
\Phi_{n}=(B C)^{\pi} \sum_{i=0}^{\infty}(B C)^{i}\left(A^{d}\right)^{2 i+2 n}+\sum_{i=0}^{\infty}\left((B C)^{d}\right)^{i+n} A^{2 i} A^{\pi}-\sum_{i=1}^{n-1}\left((B C)^{d}\right)^{i}\left(A^{d}\right)^{2 n-2 i} \\
\Psi_{n}=D^{\pi} \sum_{i=0}^{\infty} D^{2 i} C\left((B C)^{d}\right)^{i+n}+\sum_{i=0}^{\infty}\left(D^{d}\right)^{2 i+2 n} C(B C)^{i}(B C)^{\pi}-\sum_{i=1}^{n-1}\left(D^{d}\right)^{2 i} C\left((B C)^{d}\right)^{n-i} \\
\widetilde{\Sigma}_{0}=(C B)^{\pi} \sum_{i=0}^{\infty}\left(C B+D^{2}\right)^{i} C\left(A^{d}\right)^{2 i+3}+D^{\pi} \sum_{i=0}^{\infty} D^{2 i+1} C \Phi_{i+2} \\
-D^{2} \sum_{i=0}^{\infty}\left(C B+D^{2}\right)^{i} \Psi_{1}\left(A^{d}\right)^{2 i+3}+\sum_{i=0}^{\infty} \Psi_{i+2} A^{2 i+1} A^{\pi} \\
+\sum_{i=0}^{\infty}\left(D^{d}\right)^{2 i+3} C\left(A^{2}+B C\right)^{i} A^{\pi}-\sum_{i=0}^{\infty}\left(D^{d}\right)^{2 i+1} C(B C)^{i} \Phi_{1}-\Psi_{1} A^{d}
\end{gathered}
$$




$$
\begin{aligned}
\widetilde{\Lambda}= & \left((C B)^{\pi}-D^{2}\left(C B+D^{2}\right)^{d}\right) \sum_{i=0}^{\infty} \sum_{j=0}^{\infty}\left(C B+D^{2}\right)^{i} C\left(A^{d}\right)^{2 i+2 j+5}(A B+B D) D^{2 j} \\
& +D^{\pi} \sum_{i=0}^{\infty} \sum_{j=0}^{\infty} D^{2 i+1} C \Phi_{i+j+3}(A B+B D) D^{2 j} \\
& +\sum_{i=0}^{\infty} \sum_{j=0}^{i} \Psi_{i+3} A^{2 j+1}(A B+B D) D^{2 i-2 j} \\
& +\sum_{i=0}^{\infty} \sum_{j=0}^{i}\left(D^{d}\right)^{2 i+5} C\left(A^{2}+B C\right)^{j}(A B+B D) D^{2 i-2 j} \\
& -\sum_{i=0}^{\infty} \sum_{j=0}^{\infty} \Psi_{i+1} A^{2 i}\left(A^{d}\right)^{2 j+3}(A B+B D) D^{2 j} \\
& -\sum_{i=0}^{\infty} \sum_{j=0}^{\infty}\left(D^{d}\right)^{2 i+1} C\left(A^{2}+B C\right)^{i} \Phi_{j+2}(A B+B D) D^{2 j} \\
\left(C B+D^{2}\right)^{d}= & D^{\pi} \sum_{i=0}^{\infty} \sum_{j=0}^{\infty} D^{2 i}\left((C B)^{d}\right)^{i+j+1} D^{2 j}+\sum_{i=0}^{\infty}\left(D^{d}\right)^{2 i+2}(C B)^{i}(C B)^{\pi} \\
& -\sum_{i=0}^{\infty} \sum_{j=0}^{\infty}\left(D^{d}\right)^{2 i+2}(C B)^{i}\left((C B)^{d}\right)^{j+1} D^{2 j+2} \\
& +\sum_{i=0}^{\infty} \sum_{j=0}^{i}\left(D^{d}\right)^{2 i+6}(C B)^{j+1} D^{2 i-2 j+2}
\end{aligned}
$$

Proof. It is easy to see that

$$
M^{2}=\left(\begin{array}{cc}
A^{2}+B C & A B+B D \\
C A+D C & C B+D^{2}
\end{array}\right)
$$

Notice that $A B C=0$, by Lemma 2.2 we have $A^{2}+B C$ is generalized Drazin invertible and

$$
\left(A^{2}+B C\right)^{d}=(B C)^{\pi} \sum_{i=0}^{\infty}(B C)^{i}\left(A^{d}\right)^{2 i+2}+\sum_{i=0}^{\infty}\left((B C)^{d}\right)^{i+1} A^{2 i} A^{\pi} .
$$

Also $\left(A^{2}+B C\right)^{\pi}=A^{\pi}-B C\left(A^{2}+B C\right)^{d}$. By Theorem 4.2, we have $\left(C B+D^{2}\right)^{d}$ is as in (4.5) with $D$ replaced by $D^{2}$ and

$$
\begin{aligned}
\left(C B+D^{2}\right)^{d}= & D^{\pi} \sum_{i=0}^{\infty} \sum_{j=0}^{\infty} D^{2 i}\left((C B)^{d}\right)^{i+j+1} D^{2 j}+\sum_{i=0}^{\infty}\left(D^{d}\right)^{2 i+2}(C B)^{i}(C B)^{\pi} \\
& -\sum_{i=0}^{\infty} \sum_{j=0}^{\infty}\left(D^{d}\right)^{2 i+2}(C B)^{i}\left((C B)^{d}\right)^{j+1} D^{2 j+2} \\
& +\sum_{i=0}^{\infty} \sum_{j=0}^{i}\left(D^{d}\right)^{2 i+6}(C B)^{j+1} D^{2 i-2 j+2} \\
\left(C B+D^{2}\right)^{\pi}= & (C B)^{\pi}-\sum_{i=0}^{\infty}\left((C B)^{d}\right)^{i+1} D^{2 i+2}-D^{2}\left(C B+D^{2}\right)^{d} .
\end{aligned}
$$

It follows from Theorem 3.2 that

$$
\left(M^{2}\right)^{d}=\left(\begin{array}{cc}
\left(A^{2}+B C\right)^{d} & \widetilde{\Gamma} \\
\widetilde{\Sigma}_{0} & \left(C B+D^{2}\right)^{d}+\widetilde{\Lambda}
\end{array}\right),
$$


where $\widetilde{\Gamma}, \widetilde{\Sigma}_{0}$ and $\widetilde{\Lambda}$ are correspondingly $\Gamma, \Sigma_{0}$ and $\Lambda$ in Theorem 3.2 with $A, B, C, D$ replaced by $A^{2}+B C, A B+B D, C A+D C, C B+D^{2}$, respectively. Notice that $\widetilde{\Gamma} C=0$, $\widetilde{\Lambda} C=0$ and $M^{d}=\left(M^{2}\right)^{d} M$, we have

$$
M^{d}=\left(\begin{array}{cc}
\left(A^{2}+B C\right)^{d} A & \left(A^{2}+B C\right)^{d} B+\widetilde{\Gamma} D \\
\widetilde{\Sigma}_{0} A+\left(C B+D^{2}\right)^{d} C & \widetilde{\Sigma}_{0} B+\left(C B+D^{2}\right)^{d} D+\widetilde{\Lambda} D
\end{array}\right) .
$$

For any $n \geq 1$, by the hypothesis of the theorem, we have

$$
\begin{aligned}
\left(\left(A^{2}+B C\right)^{d}\right)^{n}= & (B C)^{\pi} \sum_{i=0}^{\infty}(B C)^{i}\left(A^{d}\right)^{2 i+2 n}+\sum_{i=0}^{\infty}\left((B C)^{d}\right)^{i+n} A^{2 i} A^{\pi} \\
& -\sum_{i=1}^{n-1}\left((B C)^{d}\right)^{i}\left(A^{d}\right)^{2 n-2 i} \\
\left(\left(C B+D^{2}\right)^{d}\right)^{n} C= & D^{\pi} \sum_{i=0}^{\infty} D^{2 i} C\left((B C)^{d}\right)^{i+n}+\sum_{i=0}^{\infty}\left(D^{d}\right)^{2 i+2 n} C(B C)^{i}(B C)^{\pi} \\
& -\sum_{i=1}^{n-1}\left(D^{d}\right)^{2 i} C\left((B C)^{d}\right)^{n-i},
\end{aligned}
$$

and

$$
\begin{aligned}
A\left(\left(A^{2}+B C\right)^{d}\right)^{n} & =\left(\left(A^{d}\right)^{2 n-1},\right. \\
\left(\left(C B+D^{2}\right)^{d}\right)^{n} D C & =\left(D^{d}\right)^{2 n-1} C .
\end{aligned}
$$

Let $\Phi_{n}=\left(\left(A^{2}+B C\right)^{d}\right)^{n}$ and $\Psi_{n}=\left(\left(C B+D^{2}\right)^{d}\right)^{n} C$. Using (4.11) to simplify $\widetilde{\Gamma}, \widetilde{\Sigma}_{0}$ and $\widetilde{\Lambda}$, we obtain their expressions as stated in the theorem.

The conditions of the following corollary are weaker than ones in [5, Theorem 3].

Corollary 4.3. Let $M$ be the form defined by (2.1) such that $A$ and $B C$ are generalized Drazin invertible. If $A B C=0, D C=0$ and $D$ be quasi-nilpotent, then $M$ is generalized Drazin invertible and

$$
M^{d}=\left(\begin{array}{cc}
\Phi_{1} A & \Phi_{1} B+\sum_{i=0}^{\infty} \Phi_{i+2}(A B+B D) D^{2 i+1} \\
C \Phi_{1} & \bar{\Sigma}_{0}+\sum_{i=0}^{\infty}\left((C B)^{d}\right)^{i+1} D^{2 i+1}+\bar{\Lambda} D
\end{array}\right),
$$

where where $\Phi_{i}$ are as in Theorem 4.3 and

$$
\begin{aligned}
\bar{\Sigma}_{0}= & C(B C)^{\pi} \sum_{i=0}^{\infty}(B C)^{i}\left(A^{d}\right)^{2 i+3}+\sum_{i=0}^{\infty} C\left((B C)^{d}\right)^{i+2} A^{2 i+1} A^{\pi}-(B C)^{d} A^{d} \\
\bar{\Lambda}= & C(B C)^{\pi} \sum_{i=0}^{\infty} \sum_{j=0}^{\infty}(B C)^{i}\left(A^{d}\right)^{2 i+2 j+5}(A B+B D) D^{2 j} \\
& +\sum_{i=0}^{\infty} \sum_{j=0}^{i} C\left((B C)^{d}\right)^{i+3} A^{2 j+1}(A B+B D) D^{2 i-2 j} \\
& -\sum_{i=0}^{\infty} \sum_{j=0}^{\infty} C\left((B C)^{d}\right)^{i+1} A^{2 i}\left(A^{d}\right)^{2 j+3}(A B+B D) D^{2 j}
\end{aligned}
$$

Corollary 4.4. If $A, D$ and $B C$ are generalized Drazin invertible and

$$
A B C=0 \text { and } B D=0,
$$

then $M$ is generalized Drazin invertible and

$$
M^{d}=\left(\begin{array}{cc}
\Phi_{1} A & \Phi_{1} B \\
\widetilde{\Sigma}_{0} A+\Psi_{1} & D^{d}+\widetilde{\Sigma}_{0} B
\end{array}\right),
$$


where $\Phi_{1}, \Psi_{1}$ and $\widetilde{\Sigma}_{0}$ are as in Theorem 4.3.

Proof. Obviously, if (4.12) holds, then (4.11) is satisfied. By Theorem 4.3, we have $\widetilde{\Lambda} D=0$ and

$$
\left(C B+D^{2}\right)^{d}=D^{\pi} \sum_{i=0}^{\infty} D^{2 i}\left((C B)^{d}\right)^{i+1}+\sum_{i=0}^{\infty}\left(D^{d}\right)^{2 i+2}(C B)^{i}(C B)^{\pi} .
$$

Therefore $\left(C B+D^{2}\right)^{d} D=D^{d}$.

The following corollaries can be obtained by Corollary 4.4 .

Corollary 4.5. [4] If $A, D$ and $B C$ are generalized Drazin invertible and

$$
A B C=0, B D=0 \text { and } D C=0,
$$

then $M$ is generalized Drazin invertible and

$$
M^{d}=\left(\begin{array}{cc}
\Phi_{1} A & \Phi_{1} B \\
C \Phi_{1} & D^{d}+C\left(\Phi_{1} A^{d}+(B C)^{d}\left(\Phi_{1} A-A^{d}\right)\right) B
\end{array}\right),
$$

where

$$
\Phi_{1}=(B C)^{\pi} \sum_{i=0}^{\infty}(B C)^{i}\left(A^{d}\right)^{2 i+2}+\sum_{i=0}^{\infty}\left((B C)^{d}\right)^{i+1} A^{2 i} A^{\pi} .
$$

Proof. By assumption, we compute $\Psi_{n}=\left((C B)^{d}\right)^{n} C$ for $n \geq 1$. Furthermore,

$$
\widetilde{\Sigma}_{0}=(C B)^{\pi} \sum_{i=0}^{\infty}(C B)^{i} C\left(A^{d}\right)^{2 i+3}+\sum_{i=0}^{\infty}\left((C B)^{d}\right)^{i+2} C A^{2 i+1} A^{\pi}-(C B)^{d} C A^{d} .
$$

By $(C B)^{d} C=C(B C)^{d}$, we can obtain the result.

Remark. It can be proved that all the results about generalized Drazin invertibility in the paper are still valid for Drazin invertible cases.

Acknowledgment. The authors would like to thank the anonymous referees and the editor for helpful suggestions and insightful comments that greatly improved the paper. The research is supported by the National Natural Science Foundation of China (No.11771076, No.11601014), Youth Fostering Foundation of Beihua University (No. 2017QNJJL12), Natural Science Foundation of Jilin Province of China (No. 20192475JC), Foundation of Jilin Educational Committee (No.JJKH20180333KJ), Project of Jilin Development and Reform Commission (No.2019C058-1) and China Postdoctoral Science Foundation (No.2018M632385).

\section{References}

[1] A. Ben-Israel and T.N.E. Greville, Generalized Inverses: Theory and Applications, Wiley, New York, 1974.

[2] S.L. Campbell, Singular Systems of Differential Equations I-II, Pitman, London, San Francisco, 1980.

[3] S.L. Campbell and C.D. Meyer, Generalized Inverses of Linear Transformations, Dover, New York, 1991.

[4] N. Castro-González, E. Dopazo and M.F. Matínez-Serrano, On the Drazin inverse of the sum of two operators and its application to operator matrices, J. Math. Anal. Appl. 350 (1), 207-215,2009.

[5] A.S. Cvetković and G.V. Milovanović, On Drazin inverse of operator matrices, J. Math. Anal. Appl. 375 (1), 331-335, 2011. 
[6] D.S. Cvetković-Ilić, The generalized Drazin inverse with commutativity up to a factor in a Banach algebra, Linear Algebra Appl. 431 (5), 783-791, 2009.

[7] D.S. Cvetković-Ilić, D.S. Djordjević and Y.M. Wei, Additive results for the generalized Drazin inverse in a Banach algebra, Linear Algebra Appl. 418 (1), 53-61, 2006.

[8] D.S. Cvetković-Ilić, X.J. Liu and Y.M. Wei, Some additive results for the generalized Drazin inverse in a Banach algebra, Electron. J. Linear Algebra 22, 1049- 1058, 2011.

[9] D.S. Cvetković-Ilić and Y.M. Wei, Representations for the Drazin inverse of bounded operators on Banach space, Electron. J. Linear Algebra 18, 613-627, 2009.

[10] D.S. Cvetković-Ilić and Y.M. Wei, Algebraic Properties of Generalized Inverses, Series: Developments in Mathematics, 52, Springer, 2017.

[11] C.Y. Deng, A note on the Drazin inverses with Banachiewicz-Schur forms, Appl. Math. Comput. 213 (1), 230-234, 2009.

[12] C.Y. Deng, Generalized Drazin inverses of anti-triangular block matrices, J. Math. Anal. Appl. 368 (1), 1-8, 2010.

[13] C.Y. Deng, D.S. Cvetković-Ilić and Y.M. Wei, Some results on the generalized Drazin inverse of operator matrices, Linear Multilinear Algebra 58 (4), 503-521, 2010.

[14] C.Y. Deng and Y.M. Wei, A note on the Drazin inverse of an anti-triangular matrix, Linear Algebra Appl. 431 (10), 1910-1922, 2009.

[15] C.Y. Deng and Y.M. Wei, Representations for the Drazin inverses of $2 \times 2$ blockoperator matrix with singular schur complement Linear Algebra Appl. 435 (11), 27662783, 2011.

[16] D.S. Djordjević and P.S. Stanmirović, On the generalized Drazin inverse and generalized resolvent, Czechoslovak Math. J. 51 (3), 617-634, 2001.

[17] D.S. Djordjević and Y.M. Wei, Additive results for the generalized Drazin inverse, J. Austral. Math. Soc. 73 (1), 115-125, 2002.

[18] E. Dopazo and M. F. Matínez-Serrano, Further results on the representation of the Drazin inverse of a $2 \times 2$ block matrix, Linear Algebra Appl. 432 (8), 1896-1904, 2010.

[19] M.P. Drazin, Pseudo-inverse in associative rings and semigroups, Amer. Math. Monthly 65 (7), 506-524, 1958.

[20] L. Guo and X.K. Du, Representations for the Drazin inverses of $2 \times 2$ block matrices, Appl. Math. Comput. 217 (6), 2833-2842, 2010.

[21] R.E. Harte, Spectral projections, Irish Math. Soc. Newsletter 11 (1), 10-15, 1984.

[22] R.E. Harte, Invertibility and Singularity for Bounded Linear Operators, Marcel Dekker, New York, 1988.

[23] R.E. Harte, On quasinilpotents in rings, Pan-Amer. Math. J. 1 (1), 10-16, 1991.

[24] R.E. Hartwig, and J.M. Shoaf, Group inverses and Drazin inverses of bidiagonal and triangular Toeplitz matrices, Austral J. Math. 24(A), 10-34, 1977.

[25] R.E. Hartwig, G.R. Wang and Y.M. Wei, Some additive results on Drazin inverse, Linear Algebra Appl. 322 (1), 207-217, 2010.

[26] J.J. Huang, Y.F. Shi and A. Chen, The representation of the Drazin inverses of antitriangular operator matrices based on resolvent expansions, Appl. Math. Comput. 242 (1), 196-201, 2014.

[27] J.J. Koliha, A generalized Drazin inverse, Glasgow Math. J. 38 (3), 367-381, 1996.

[28] J.J. Koliha, The Drazin and Moore-Penrose inverse in $C^{*}$-algebras, Math. Proc. R. Ir. Acad. 99A (1), 17-27, 1999.

[29] J.J. Koliha, D.S. Cvetković-Ilić and C. Y. Deng, Generalized Drazin invertibility of combinations of idempotents, Linear Algebra Appl. 437 (9), 2317-2324, 2012.

[30] J. Ljubisavljević and D.S. Cvetković-Ilić, Additive results for the Drazin inverse of block matrices and applications, J. Comput. Appl. Math. 235 (12), 3683-3690, 2011.

[31] C.D. Meyer and N.J. Rose, The index and the Drazin inverse of block triangular matrices, SIAM J. Appl. Math. 33 (1), 1-7, 1977.

[32] G.J. Murphy, $C^{*}$-Algebras and Operator Theory, Academic Press, San Diego, 1990. 
[33] V. Müller, Spectral theory of linear operators and spectral systems in Banach algebras, Operator Theory, Advances and Applications, 139, Birkhäuser Verlag, Basel-BostonBerlin, 2007.

[34] H. Yang and X.J. Liu, The Drazin inverse of the sum of two matrices and its applications, J. Comput. Appl. Math. 235 (5), 1412-1417, 2011.

[35] G.F. Zhuang, J.L. Chen, D.S. Cvetković-Ilić and Y.M. Wei, Additive property of Drazin invertibility of elements in a ring, Linear Multilinear Algebra 60 (8), 903-910, 2012.

[36] H.L. Zou, J.L. Chen and D. Mosić, The Drazin invertibility of an anti-triangular matrix over a ring, Stud. Sci. Math. Hung. 54 (4), 489-508, 2017.

[37] H. L. Zou, D. Mosić and J. L. Chen, The existence and representation of the Drazin inverse of a $2 \times 2$ block matrix over a ring, J. Algebra Appl., 18 (11), 2019, doi: 10.1142/S0219498819502128. 